\title{
sciendo
}

\section{Cancer Stem Cells in Head and Neck Squamous Cell Carcinoma- Treatment Modalities}

\begin{abstract}
SUMMARY
Head and neck squamous cell carcinoma (HNSCC) belongs to the most frequent cancer subtypes in the world. Mutations due to genetic and chromosomal instability, syndromes such as Fanconi anemia and the Bloom syndrome, environmental risk factors such as tobacco smoking, alcohol and human papillomavirus infection (HPV) subtypes 16,18,31,33,35,52,58 are implicated in its pathogenesis. The HNSCC belongs to the solid tumors of epithelial origin and consists of stromal, inflammatory, cancer cells and most importantly a fraction of them, the cancer stem cells (CSCs). The identification of the CSCs through their biomarkers such as CD44, CD10, CD166, CD133, CD271, ALDH, Oct4, Nanog, Sox2 and Bmil, the maintenance of their subpopulation through epithelial to mesenchymal transition, the role of HPV infection regarding their prognosis and of their microenvironment regarding their resistance to therapy, all constitute key elements that must be taken thoroughly into consideration in order to develop an effective targeted therapy. There are already therapies in place targeting specific related biomarkers, important biochemical pathways and growth factors. The aim of this literature review is to illustrate the treatment modalities available against the cancer stem cells of head and neck squamous cell carcinoma.
\end{abstract}

Key Words: Cancer Stem Cells, Oral Cancer, Oral Cancer Stem Cells, Head and Neck Cancer, HPV and Cancer, Cancer Stem Cells Treatment

\author{
Vasileios Zisis, Maria Venou, Athanasios \\ Poulopoulos, Dimitrios Andreadis
}

Department of Oral Medicine/Pathology, School of Dentistry, Aristotle University of Thessaloniki, Greece

\section{REVIEW PAPER (RP)}

Balk J Dent Med, 2021;73-79

\section{Introduction}

The prevalence of head and neck squamous cell carcinoma (HNSCC) classifies it as the $5^{\text {th }}$ more frequent cancer in the world, most probably due to its inherent invasiveness and proximity to structures of critical importance, with an overall incidence of over 300.000600.000 cases per year ${ }^{1-8}$. It consists an epithelial tumor, affecting the oral cavity, pharynx and larynx. The pathogenesis involves both environmental factors and mutations due to genetic instability. The environmental risk factors include tobacco smoking, alcohol and human papillomavirus infection (HPV) subtypes $16,18,31,33,35,52,58^{2,4,6,9}$. The genetic risk factors include chromosomal instability and syndromes such as Fanconi anemia and the Bloom syndrome ${ }^{4}$.
The HNSCCs are solid tumors with heterogeneous content, including cancer cells, stromal cells, and inflammatory cells ${ }^{2,3}$. The cancer cells originate from stem cells (characterized by self-renewal ability), from progenitor cells (which are abundant in adult tissue and have partial ability to self-renew) and from de-differentiated cells (they de-differentiate in order to convert to stem cells, this process is mediated by the phenomenon of Epithelial-Mesenchymal Transition $(\mathrm{EMT}))^{10}$. As a result, there are only 2 possible models for further cancer development: either random oncogenic mutations leading to the formation of neoplasia through cancer cells $\mathrm{s}^{6,11}$ or a distinct subpopulation of cancer stem cells (CSCs) on top of the cancer cell hierarchy (characterized by symmetrical and asymmetrical division following the pattern of normal stem cells) initiates them. The first model is the Stochastic whereas the second 
one is the Hierarchical. In the case of HNSCC, research findings support the cancer stem cell theory.

The key features of $\mathrm{CSCs}^{12}$ are the following:

1. Only a small fraction of the cancer cells within a tumor have tumorigenic potential when transplanted into immunodeficient mice.

2. The CSC subpopulation can be separated from the other cancer cells by distinct surface markers.

3. Tumors resulting from the CSCs contain the mixed tumorigenic and nontumorigenic cells of the original tumor.

4. The CSC subpopulation can be serially transplanted through multiple generations, indicating that it is a self-renewing population.

A pluripotent cell subpopulation featuring stemness, self-renewal and oncogenic properties, has the ability to generate, maintain and drive the whole volume of the heterogeneous tumor ${ }^{11,13}$. On some level, tumor cells persist on normal differentiation, leading to disturbed maturation and heterogeneous histological sections. As a result, certain products are present in HNSCC creating the keratinization. Regarding the theory of "field cancerization" which is associated with cancer recurrences and development of secondary tumor, molecular alterations which appear to the cells of the mucous membrane adjacent to the tumor are driven by the presence of CSCs either in a polyclonal or monoclonal pattern ${ }^{14}$. On that note, apart from molecular and genetic changes in normal mucosa, putative CSCs are also found in seemingly tumor free surgical margins of oral cancer ${ }^{15}$.

The role of CSCs in oral squamous cell carcinoma (OSCC) is well established. They may originate from the local basal layer, vessel walls, blood, muscle tissue, adipose tissue, the cell fusion between hematopoietic stem cells and oral keratinocytes, and finally EMT de-differentiated cells ${ }^{10}$.

Treatment modalities have consistently improved. Most patients receive primary radio chemotherapy as this appears to be the most effective treatment modality in relation with the overall survival rates and the control of loco-regional recurrences and distant metastasis. The prognosis remains poor. The 5-year overall survival (OS) rate is $50 \%^{6}$, when regarding the frequency of cancer induced mortality HNCSS is the 7 th most common malignancy ${ }^{5}$. The low survival rate is attributed to late diagnosis ${ }^{16}$, local-regional relapses (10-50\% of the patients $)^{5,6,17}$, development of second primary tumor $(2-30 \% \text { of the patients })^{5}$, lymph node and distant metastasis $(25 \%)^{14,16,18}$ and radio- and chemoresistance $^{14,16}$.

Suboptimal control of loco- regional recurrences has been associated with multiple factors, including the volume of the tumor and hypoxia-induced alterations: A more voluminous tumor is considered to have a larger number of CSCs while hypoxia causes through multiple mechanisms a reduction in tumor radiosensitivity ${ }^{19}$, enhancing the survival of malignant cells ${ }^{5}$. Especially OSCC relies on hypoxia cellular response ${ }^{10}$. This means that hypoxia leads not only to direct resistance to conventional treatment but also to cellular phenotypic alterations creating an ever more resilient CSCs' subpopulation $^{5}$.

\section{Material and Methods}

This review aims to investigate the role of HNSCCCSCs regarding tumor pathogenesis as well as current and experimental treatment modalities. An electronic search of the literature was performed between July 2019 and February 2020 to identify articles investigating HNSCC, CSCs and treatment options. The literature search was conducted using MEDLINE (National Library of Medicine)-PubMed without restrictions concerning the date of publication. The following keywords were used (connecting different keywords with AND, OR): head and neck squamous cell carcinoma, oral squamous cell carcinoma, cancer stem cells and targeted therapies. This was followed by a manual search and references were used to identify relevant articles. The articles identified from the electronic and manual search were screened to eliminate those that failed to meet the respective inclusion and exclusion criteria as listed below.

Inclusion criteria for analysis of human and animal studies:

- Randomized controlled clinical trials, prospective and retrospective clinical studies, Case reports or case studies, Animal studies that evaluated the pathogenesis of HNSCCs, the HNSCSCs' implication and relevant treatment regimes.

Exclusion criteria for analysis of human and animal studies:

- Studies in a language other than English or without an English abstract.

After this screening 41 studies remained for full text analysis.

\section{Results}

Identification of CSCs' subpopulations and clinical importance of the respective CSCs' biomarkers. The identification of the subpopulations of CSCs is immensely important since it could lead to improved treatment modalities. Every CSC subpopulation expresses different markers in accordance with the tissue of origin. Flow cytometry/fluorescence-activated cell sorting (FACS) is the best technique so far for the identification and isolation of $\mathrm{CSCs}^{20}$ although MACS has proven itself as a different and in some experiments, more convenient 
method for cell isolation ${ }^{21}$. The most reliable marker so far is CD44 and its isoforms. CD44v3 is associated with advanced $\mathrm{T}$ stage and regional metastasis. CD44v6 is associated with advanced $\mathrm{T}$ stage, perineural invasion and shorter disease-free survival. CD44v10 is associated with distant metastasis, radiation failure and shorter disease-free survival ${ }^{22}$. CD44v9 specifically enhances the defense mechanism against ROS. CD44 in general correlates with tumor-sphere formation and resistance to chemotherapeutics. The combination of CD44 with the marker CD24 proves to be even better at isolating $\mathrm{CSCs}^{20}$. The CD10 marker enhances the stem cell proliferation and tumor-sphere formation. Its level increases after RT or CT treatment. Its expression associates with recurrence and metastases. The CD166 marker associates with larger tumors and localization at the tumor invasive front. The CD133 marker correlates with increased proliferation, EMT phenotype, tumor-sphere formation, self-renewal and in-vivo tumorigenicity. The combination of both positive CD133 and CD44 showed more malignant features compared to only CD133 or CD44 expression ${ }^{23}$. The CD271 marker correlates with high tumorigenicity and localization at the tumor invasive front. The ALDH marker is involved in EMT, self-renewal, tumor formation, and resistance to chemotherapeutics. Its ALDH1A1 isoform correlates strongly with enhanced ALDH activity ${ }^{20}$. The markers Oct4, Nanog and Sox 2 are associated with EMT and metastasis. Finally, the Bmil marker is associated with HNSCC and especially the oral squamous cell carcinoma ${ }^{24}$ (Table 1).

Table 1. Biomarkers of cancer stem cells (CSCs)

\begin{tabular}{|c|c|}
\hline Biomarker & Associations \\
\hline CD44 & $\begin{array}{l}\text { tumor-sphere formation and resistance to } \\
\text { chemotherapeutics }\end{array}$ \\
\hline CD44v3 & advanced $\mathrm{T}$ stage, regional metastasis \\
\hline CD44v6 & $\begin{array}{l}\text { advanced T stage, perineural invasion, shorter } \\
\text { disease-free survival }\end{array}$ \\
\hline CD44v9 & ROS defense mechanism \\
\hline CD44v10 & $\begin{array}{l}\text { shorter disease-free survival, radiation failure, } \\
\text { distant metastasis }\end{array}$ \\
\hline CD10 & stem cell proliferation and tumor-sphere formation \\
\hline CD166 & $\begin{array}{l}\text { larger tumors and localization at the tumor invasive } \\
\text { front }\end{array}$ \\
\hline CD133 & $\begin{array}{l}\text { increased proliferation, EMT phenotype, tumor- } \\
\text { sphere formation, self-renewal and in-vivo } \\
\text { tumorigenicity }\end{array}$ \\
\hline $\mathrm{CD} 271$ & $\begin{array}{l}\text { high tumorigenicity and localization at the tumor } \\
\text { invasive front }\end{array}$ \\
\hline ALDH & $\begin{array}{l}\text { EMT, self-renewal abilities, tumor formation, and } \\
\text { resistance to chemotherapeutics }\end{array}$ \\
\hline Oct4 & EMT and cancer metastasis \\
\hline Nanog & EMT and cancer metastasis \\
\hline Sox 2 & EMT and cancer metastasis \\
\hline Bmi1 & head and neck tumorigenesis \\
\hline
\end{tabular}

\section{Maintenance of CSCs' subpopulation through EMT}

Epithelial-to-mesenchymal transition (EMT), a phenomenon that takes place mostly during development, epithelial cells acquire a mesenchymal phenotype associated with tumor growth and metastasis due to enhanced motility and invasiveness. This invasive phenotype allows them to gain easier access to the lymphatic, venous and/or arterial circulation ${ }^{3}$. Phenotypic plasticity seems to be a key component of EMT, as CSCs with this characteristic are considered more resistant to chemotherapeutic agents ${ }^{6,25}$. Thus, the transformed epithelial cells obtain mesenchymal traits, enabling them to cross endothelial barriers and enter blood and lymphatic circulations, thus contributing to metastasis. When the respective tissue is reached, the stimuli that led to the initial transition cease and the metastasized cells invert to their original epithelial phenotype via mesenchymal-epithelial transition ${ }^{26}$. Multiple signal pathways are considered to be implicated in EMT such as Notch, TGF- $\beta$, Hedgehog and the Wnt $/ \beta$-catenin signaling ${ }^{26}$. Transcription factors involved in the CSCs' gene expression, directly and indirectly leading to the aforementioned EMT seem to sustain the survival of CSCs subpopulations ${ }^{26}$.

\section{Resistance to therapy and CSCs microenvironment}

The characteristics of CSCs lead to resistance to any conventional treatment protocolls. The radioresistance is related to self-renewal capacity, DNA repair capacity, free-radical scavenging, upregulation of cell cycle control mechanisms and specific interactions with the stromal microenvironment. The resistance to chemotherapy is attributed to accelerated drug removal e.g. due to upregulated efflux pumps and drug metabolism ${ }^{3}$.

Hypoxia is a major micro-environmental influence. Initially, as the HNSCC grows, blood supply does not suffice and areas of hypoxia develop ${ }^{5}$. Hypoxia-inducible factor 1-alpha (HIF- 1a) decreases the response to radiotherapy through suppression of the production of ROS, a prerequisite for an effective radiation treatment ${ }^{5}$. HIF- 1a also supports the migration and the invasive growth of cancer as well as the EMT process (possibly through the loss of cell adhesion, increased cell motility and production of proteinases) $)^{5}$. HIF- 1a may also assist the cell adaptation to hypoxic stress through the upregulation of genes such as the glucose transporter 1 (GLUT1) and the vascular endothelial growth factor (VEGF). Thus, the maintenance of the stem cells niche stabilizes $^{1}$. Regarding current therapeutic regimens, HIF1a supports the resistance to drug-induced senescence in cancer cell lines 5 . Unfortunately most of the therapy regimens focus on the bulk tumor mass ${ }^{27}$, meaning that they focus on successfully inducing hypoxia to prevent further growth, accidentally increasing the CSC fraction 
of tumors through the role of HIF- $1 \mathrm{a}^{5}$. This fraction further aids the post-treatment repopulation of cancer cells, leaving behind the assassin CSCs that serve as a reservoir for tumor repopulation post-therapy.

Regarding possible correlation to CSCs' biomarkers, increased CD44 levels are associated with tumor hypoxia $^{28}$ through HIF-1a induced upregulation of the CD44 gene expression ${ }^{29}$. Another pathway of critical importance is the MET. The transcription of the MET proto-oncogene is partially triggered by hypoxia. The MET pathway promotes the self-renewal and tumorigenicity in HNSCC stem cells ${ }^{17}$. The pharmacologic selective inhibition of MET leads to the elimination of $\mathrm{CSCs}^{30}$. Therefore, the MET inhibition prevents hypoxia-induced cell growth and a link between hypoxic areas and MET overexpression is established ${ }^{31}$.

The CSCs depend on their microenvironment for subsistence and self-maintenance. Most of the stem cells are located close to blood vessels since the endothelial cells secrete factors that promote the selfrenewal and survival and provide nutrients and oxygen. The components (stromal cells, inflammatory cells and vessels) of the niche in general provide the signals that maintain CSCs in their undifferentiated state. Therefore, a key factor regarding therapeutic resistance is the complex interaction between the neoplasia and various niche components ${ }^{10}$. Thus, targeting the stem cell niche or tissue compounds diminishes the nutrition and negates the signaling needed by CSCs to proliferate ${ }^{3}$.

The resistance to therapy is further reinforced by the general immunosuppression induced by cancer. Increased apoptosis of tumor-specific CD8+ T-cells and increased tumor infiltrating $\mathrm{T}$ regulatory cells have been demonstrated. Elevated IL-6 levels could independently predict tumor recurrence, poor survival, and tumor metastasis. Initially, CSCs can be destroyed by the immune system but after a certain point equilibrium is reached where cells with non-immunogenic phenotype survive the initial termination process, escape and prevail through constant growth. This evasion of the immune mediated destruction is manifold. To name but a few, lack of expression of known differentiation antigens or $\mathrm{MHC}$ molecules, lack of co-stimulatory ligands, expression of co-inhibitory ligands as well as expression of inhibitory cytokines, all play a critical role in surviving the initial phase of extermination ${ }^{16}$.

\section{HPV and HNSCC}

Human papilloma virus is a well-established causative factor of HNSCC and more specifically oropharyngeal cancer with distinct epidemiologic and prognostic characteristics. Over 160 genotypes of HPV have been found, including the high- risk oncogenic subtypes (a- HPV genus). The most important HPVrelated cancer subtypes ${ }^{4,19,32}$ appear to be subtypes 16 and 18. More specifically, HPV interacts with cell surface receptors, abundant in basal cells and epithelial stem cells, and in malignant tumor the virus integrated into the genome of the host. From the 8 viral proteins, encoded by the viral genome, E6 and E7 oncoproteins seem to be responsible for changes related to cell proliferation. The presence of HPV causes chromosomal anomalies and cellular immortalization ${ }^{4}$. Linge et al. point out that the presence of HPV consists a positive prognostic factor for the control of loco-regional recurrence. Moreover, the investigators refer that the expression of stemness biomarkers has been found to be decreased in HPV positive tumors and HPV16 positive tumors appear to be more radiosensitive at the same time. Nevertheless, treatment remains suboptimal for patients with HPV positive cancer, the exact reasons remain unclear $^{3}$. Smoking seems to be associated with increased EGFR expression that leads to a poor prognosis in comparison with other HPV positive tumors ${ }^{33}$. However, the unanswered question is why HPV+ tumors are considered to generally have a better prognosis when treated with chemoradiation compared to non-HPV. In HPV+ HNSCC, although E6 and E7 genes immortalize infected cells, field cancerization is not present. This lack of a genetically unstable area provides genomically speaking a much more homogenous cellular genotype, thus facilitating a better prognosis. The immune mediated destruction of HPV infected cells and the fact that P53 and pRb are rendered dormant but not silenced via E6 and E7 genes are also regarded as positive prognostic markers. On the other hand, the immune response (defined in this case as antibody production against the highly expressed p16 in HPV+ HNSCC) is not significantly strong ${ }^{32}$. Regarding the role of EGFR, its activity is essential for maintaining cancer stem-like state while also interacting with CD44 to manifest chemoresistance. This interaction is not documented in HPV+ tumors, partially explaining why they respond better to chemotherapy ${ }^{32}$. According to Tang et al, the recurrences of HPV positive tumors have a relationship with CSCs and a probable poor prognosis can be attributed to the presence of increased EGFR expression and $\mathrm{ALDH}^{33}$. In literature, studies that investigate the relationship between CSCs and HPV present equivocal findings. Thus, the effect of E6 on CSCs remains poorly understood. It has been speculated that HPV positive cancers contain fewer CSCs, conferring a better prognosis and less treatment resistance 4 . Finally, two closing remarks regarding independent but nonetheless noteworthy findings: there are no clear results about how resistant HPV- positive tumors are to cisplatin therapy ${ }^{4}$. The immunostaining for p16 protein is a surrogate marker for HPVrelated oropharyngeal carcinomas due to the positive correlation observed between HPV detection and p16 overexpression $^{2}$. 


\section{HNSCCs' targeted therapy}

An ideal treatment would preferentially target specific CSCs' subpopulations which are responsible for the tumor expansion, thus establishing a patientspecific therapy with minimal adverse effects. The combination of Cisplatin and PTC-209 dramatically reduced $\mathrm{Bmil}^{+}$tumor cells after 5 days, thus successfully targeting $\mathrm{Bmil}^{+}$tumors $^{34}$. 5T4 shows a high expression pattern in head and neck CSCs $\left(\mathrm{CD}_{4} 4^{+} / \mathrm{ALDH}^{+}\right)$. The Anti-5T4 antibody-drug MEDI0641 reduces the fraction of those CSCs and prevents local recurrence ${ }^{35}$. The therapeutic agent BGJ398 dramatically reduced cisplatin-resistant $\mathrm{CD}_{4}{ }^{+} / \mathrm{ALDH}^{+}$cells by inhibiting FGF receptors (suggesting that FGF confers at least partially a resistance to cisplatin) ${ }^{36}$. Loss of function mutations of Notch lead to upregulation of the Wnt signaling (since Notch suppresses Wnt signaling). HNSCC lines, responsive to treatment by LGK974, are enriched by LoF Notch1 mutations. LGK974 exhibits after a mere 2 weeks post treatment strong inhibition of tumor growth ${ }^{37}$. ALDH1 is upregulated in HNSCC tumors following exposure to cisplatin (suggesting that ALDH1 also mediates a partial cisplatin resistance). ALDH1 induction's inhibition through Aldi-6 leads to a reduction of cell survival ${ }^{38}$. Selective inhibitor PF-2341066 of the c- MET downregulates the Wnt $/ \beta$ catenin signaling through the FZD8 receptor, thus eliminating the $\mathrm{HNSCCs}^{30}$. Both $\mathrm{CD} 44$ and its CD44v6 subtype constitute main important targets. The radionuclide 186Re-cmAb (U36) targets CD44 in general ${ }^{39}$ while the anti-CD44v6 antibody BIWA enables the intraoperative imaging of tumor borders and remnants, invasion zone as well as the microscopic mapping of the tumor lesions ${ }^{40}$. Nivolumab and pembrolizumab, both anti-PD1 antibodies, improve the overall survival of HNSCC compared to traditional chemotherapy as a second line therapy ${ }^{41}$ (Table 2).

Table 2. Targeting specific molecules for oral cancer management

\begin{tabular}{ll}
\hline Target & Therapy \\
\hline Bmi1 $^{+}$ & PTC-209 \\
5T4 & MEDI0641 \\
FGF & BGJ398 \\
Porcupine (PORCN) (Wnt & LGK974 \\
signaling) & Aldi-6 \\
ALDH1 & PF-2341066 \\
cMET/FZD8 & Radionuclide ${ }^{186}$ Re-cmAb \\
CD44 & (U36) \\
& Anti-CD44v6 antibody \\
CD44v6 & BIWA \\
\hline
\end{tabular}

\section{Conclusions}

A multitude of targeted therapies are already in the phase of clinical trials. The future looks promising as stem cell research works diligently at identifying more diagnostically and prognostically significant biomarkers and unraveling the still elusive pathomechanisms behind epithelial to mesenchymal transition, the biochemical pathways which allow cancer stem cells to metastasize, propagate and prevail as well as the exact nature and characteristics of their niche. In the not so distant future treatment protocols are bound to become even more selective, thus achieving the ideal combination of patient-selective therapy whilst keeping major and minor adverse effects at the bare minimum. Whether these protocols will consist of monoclonal antibodies, small molecule inhibitors, more exotic entities such as antisense oligonucleotides or a combination of them all remains to be seen.

\section{References}

1. Ketkaew Y, Osathanon T, Pavasant P, Sooampon S. Apigenin inhibited hypoxia induced stem cell marker expression in a head and neck squamous cell carcinoma cell line. Arch Oral Biol, 2017;74:69-74.

2. de Moraes FPP, Lourenço SV, Ianez RCF, de Sousa EA, Silva MM da C, Damascena AS, et al. Expression of stem cell markers in oral cavity and oropharynx squamous cell carcinoma. Oral Surg Oral Med Oral Pathol Oral Radiol, 2017;123:113-122.

3. Méry B, Guy JB, Espenel S, Wozny AS, Simonet S, Vallard A, et al. Targeting head and neck tumoral stem cells: From biological aspects to therapeutic perspectives. World J Stem Cells, 2016;8:13-21.

4. Pullos AN, Castilho RM, Squarize CH. HPV infection of the head and neck region and its stem cells. J Dent Res, 2015;94:1532-1543.

5. Gammon L, Mackenzie IC. Roles of hypoxia, stem cells and epithelial-mesenchymal transition in the spread and treatment resistance of head and neck cancer. J Oral Pathol Med, 2016;45:77-82.

6. Kaseb HO, Lewis DW, Saunders WS, Gollin SM. Cell division patterns and chromosomal segregation defects in oral cancer stem cells. Genes Chromosomes Cancer, 2016;55:694-709.

7. Biddle A, Gammon L, Liang X, Costea DE, Mackenzie IC. Phenotypic Plasticity Determines Cancer Stem Cell Therapeutic Resistance in Oral Squamous Cell Carcinoma. EBioMedicine, 2016;4:138-145.

8. Yoshihama R, Yamaguchi K, Imajyo I, Mine M, Hiyake $\mathrm{N}$, Akimoto N, et al. Expression levels of SOX2, KLF4 and brachyury transcription factors are associated with metastasis and poor prognosis in oral squamous cell carcinoma. Oncol Lett, 2016;11:1435-1446.

9. López-Lázaro M. A local mechanism by which alcohol consumption causes cancer. Oral Oncol, 2016;62:149-152. 
10. Moharil R, Dive A, Khandekar S, Bodhade A. Cancer stem cells: An insight. J Oral Maxillofac Pathol, 2017;21:463.

11. Kaseb HO, Fohrer-Ting H, Lewis DW, Lagasse E, Gollin SM. Identification, expansion and characterization of cancer cells with stem cell properties from head and neck squamous cell carcinomas. Exp Cell Res, 2016;348:75-86.

12. Prince MEP, Ailles LE. Cancer stem cells in head and neck squamous cell cancer. J Clin Oncol, 2008;26:2871-2875.

13. Gemenetzidis E, Gammon L, Biddle A, Emich H, Mackenzie IC. Invasive oral cancer stem cells display resistance to ionising radiation. Oncotarget, 2015;6:4396443977.

14. Simple M, Suresh A, Das D, Kuriakose MA. Cancer stem cells and field cancerization of Oral squamous cell carcinoma. Oral Oncol, 2015;51:643-651.

15. Lazarevic M, Milosevic M, Trisic D, Toljic B, Simonovic J, Nikolic N, et al. Putative cancer stem cells are present in surgical margins of oral squamous cell carcinoma. JBUON, 2018;23:1686-1692.

16. Prince MEP, Zhou L, Moyer JS, Tao H, Lu L, Owen J, et al. Evaluation of the immunogenicity of ALDHhigh human head and neck squamous cell carcinoma cancer stem cells in vitro. Oral Oncol, 2016;59:30-42.

17. Lim YC, Kang HJ, Moon JH. C-Met pathway promotes self-renewal and tumorigenecity of head and neck squamous cell carcinoma stem-like cell. Oral Oncol, 2014;50:633-639.

18. Peng CY, Liao YW, Lu MY, Yu CH, Yu CC, Chou MY. Downregulation of miR-1 enhances tumorigenicity and invasiveness in oral squamous cell carcinomas. J Formos Med Assoc, 2017;116:782-789.

19. Linge A, Lohaus F, Löck S, Nowak A, Gudziol V, Valentini C, et al. HPV status, cancer stem cell marker expression, hypoxia gene signatures and tumour volume identify good prognosis subgroups in patients with HNSCC after primary radiochemotherapy: A multicentre retrospective study of the German Cancer Consortium Radiation Oncology Group (DKTK-ROG). Radiother Oncol, 2016;121:364373.

20. Elkashty OA, Ashry R, Tran SD. Head and neck cancer management and cancer stem cells implication. Saudi Dent J, 2019;31:395-416.

21. Lee MY, Lufkin T. Development of the "three-step macs": A novel strategy for isolating rare cell populations in the absence of known cell surface markers from complex animal tissue. J Biomol Tech, 2012;23:69-77.

22. Wang SJ, Wong G, de Heer AM, Xia W, Bourguignon LYW. CD44 Variant isoforms in head and neck squamous cell carcinoma progression. Laryngoscope, 2009;119:1518-1530.

23. Wang J, Wu Y, Gao W, Li F, Bo Y, Zhu M, et al. Identification and characterization of CD133 + CD44 + cancer stem cells from human laryngeal squamous cell carcinoma cell lines. J Cancer, 2017;8:497-506.

24. Liu W, Feng JQ, Shen XM, Wang HY, Liu Y, Zhou ZT. Two stem cell markers, ATP-binding cassette, G2 subfamily (ABCG2) and BMI-1, predict the transformation of oral leukoplakia to cancer: A long-term follow-up study. Cancer, 2012;118:1693-1700.
25. Sun S, Wang Z. Head neck squamous cell carcinoma c-Met + cells display cancer stem cell properties and are responsible for cisplatin-resistance and metastasis. Int $\mathrm{J}$ Cancer, 2011;129:2337-2348.

26. Wang SS, Jiang J, Liang XH, Tang YL. Links between cancer stem cells and epithelial- mesenchymal transition. Onco Targets Ther, 2015;8:2973-2980.

27. Naik PP, Das DN, Panda PK, Mukhopadhyay S, Sinha $\mathrm{N}$, Praharaj PP, et al. Implications of cancer stem cells in developing therapeutic resistance in oral cancer. Oral Oncol, 2016;62:122-135.

28. Linge A, Lock S, Gudziol V, Nowak A, Lohaus F, Von Neubeck C, et al. Low cancer stem cell marker expression and low hypoxia identify good prognosis subgroups in HPV(-) HNSCC after postoperative radiochemotherapy: A multicenter study of the DKTK-ROG. Clin Cancer Res, 2016;22:2639-2649.

29. Liang G, Li S, Du W, Ke Q, Cai J, Yang J. Hypoxia regulates CD44 expression via hypoxia-inducible factor- $1 \alpha$ in human gastric cancer cells. Oncol Lett, 2017;13:967972.

30. Sun S, Liu S, Duan SZ, Zhang L, Zhou H, Hu Y, et al. Targeting the c-Met/FZD8 signaling axis eliminates patient-derived cancer stem-like cells in head and neck squamous carcinomas. Cancer Res, 2014;74:7546-7559.

31. Pennacchietti S, Michieli P, Galluzzo M, Mazzone M, Giordano S, Comoglio PM. Hypoxia promotes invasive growth by transcriptional activation of the met protooncogene. Cancer Cell, 2003;3:347-361.

32. Modur V, Thomas-Robbins K, Rao K. HPV and CSC in HNSCC cisplatin resistance. Front Biosci (Elite ed), 2015;7:58-66.

33. Tang AL, Hauff SJ, Owen JH, Graham MP, Czerwinski MJ, Park JJ, et al. UM-SCC-104: A New human papillomavirus-16-positive cancer stem cell-containing head and neck squamous cell carcinoma cell line. Head Neck, 2012;34:1480-1491.

34. Chen D, Wu M, Li Y, Chang I, Yuan Q, Ekimyan-Salvo M, et al. Targeting BMI1+ Cancer Stem Cells Overcomes Chemoresistance and Inhibits Metastases in Squamous Cell Carcinoma. Cell Stem Cell, 2017;20:621-634.

35. Kerk SA, Finkel KA, Pearson AT, Warner KA, Zhang Z, Nör F, et al. 5T4-targeted therapy ablates cancer stem cells and prevents recurrence of head and neck squamous cell carcinoma. Clin Cancer Res, 2017;23:2516-2527.

36. McDermott SC, Rodriguez-Ramirez C, McDermott SP, Wicha MS, Nör JE. FGFR signaling regulates resistance of head and neck cancer stem cells to cisplatin. Oncotarget, 2018;9:25148-25165.

37. Liu J, Pan S, Hsieh MH, Ng N, Sun F, Wang T, et al. Targeting Wnt-driven cancer through the inhibition of Porcupine by LGK974. Proc Natl Acad Sci U S A, 2013;110:20224-20249.

38. Kim J, Shin JH, Chen C-H, Cruz L, Farnebo L, Yang J, et al. Targeting aldehyde dehydrogenase activity in head and neck squamous cell carcinoma with a novel small molecule inhibitor. Oncotarget, 2017;8:52345-52356.

39. Peitzsch C, Nathansen J, Schniewind SI, Schwarz F, Dubrovska A. Cancer stem cells in head and neck squamous cell carcinoma: Identification, characterization and clinical implications. Cancers (Basel), 2019;11:616. 
40. Odenthal J, Rijpkema M, Bos D, Wagena E, Croes H, Grenman R, et al. Targeting CD44v6 for fluorescenceguided surgery in head and neck squamous cell carcinoma. Sci Rep, 2018;8:10467.

41. Chen D, Wang CY. Targeting cancer stem cells in squamous cell carcinoma. Precis Clin Med, 2019;2:152165.

Conflict of Interests: Nothing to declare.

Financial Disclosure Statement: Nothing to declare.

Human Rights Statement: None required.

Animal Rights Statement: None required.
Received on July 16, 2020.

Revised on August 8, 2020.

Accepted on September 22, 2020.

Correspondence:

Zisis Vasileios

Department of Oral Medicine/Pathology

School of Dentistry, Thessaloniki, Greece

e-mail:zisisdent@gmail.com 